\title{
Active Damping of the E-P Instability at the Los Alamos Proton Storage Ring
}

\author{
R. J. Macek ${ }^{\mathrm{a})}$ \\ Los Alamos National Laboratory, Los Alamos, New Mexico 87545, USA \\ S. Assadi \\ Oak Ridge National Laboratory, Oak Ridge, Tennessee 37831, USA \\ J. M. Byrd \\ Lawrence Berkeley National Laboratory, One Cyclotron Road, Berkeley, California 94720, USA \\ C. E. Deibele and S. D. Henderson \\ Oak Ridge National Laboratory, Oak Ridge, Tennessee 37831, USA
}

S. Y. Lee

Indiana University, Bloomington, Indiana 47405, USA

R. C. McCrady

Los Alamos National Laboratory, Los Alamos, New Mexico 87545, USA

M. F. T. Pivi

Stanford Linear Accelerator Center, Menlo Park, California 94025, USA

M. A. Plum

Oak Ridge National Laboratory, Oak Ridge, Tennessee 37831, USA

S. B. Walbridge

Indiana University, Bloomington, Indiana 47405, USA

T. J. Zaugg

Los Alamos National Laboratory, Los Alamos, New Mexico 87545, USA

\begin{abstract}
A prototype of an analog, transverse (vertical) feedback system for active damping of the two-stream (e-p) instability has been developed and successfully tested at the Los Alamos Proton Storage Ring (PSR). This system was able to improve the instability threshold by approximately $30 \%$ (as measured by the change in RF buncher voltage at instability threshold). The feedback system configuration, setup procedures, and optimization of performance are described. Results of several experimental tests of system performance are presented including observations of instability threshold improvement and grow-damp experiments, which yield estimates of instability growth and damping rates. A major effort was undertaken to identify and study several factors limiting system performance. Evidence obtained from these tests suggests that performance of the prototype was limited by higher instability growth rates arising from beam leakage into the gap at lower RF buncher voltage and the onset of instability in the horizontal plane, which had no feedback.
\end{abstract}

\section{INTRODUCTION}

High luminosity colliders for nuclear and particle physics, drivers for neutron sources, high intensity muon and neutrino beams, plus accelerator driven power generation and nuclear waste transmutation are among the many applications of high intensity ions beams. Beam intensity in these applications is often limited by beam instabilities caused by space charge forces, impedances of accelerator components, and noise. In the last decade, the electron cloud associated with intense positive ion beams has produced a number of accelerator performance-limiting effects, including the elec-

${ }^{a)}$ Electronic mail: macek@lanl.gov or rjmacek@comcast.net. tron cloud instability, ${ }^{1-4}$ which in many ways resembles the two-stream instability in plasmas, ${ }^{5}$ particularly for long bunch machines.

For high energy colliders with short bunches, the instability can often be alleviated by changing the bunch spacing to suppress resonance formation of an electron cloud, and/or by introducing a bunch-train gap to dissipate the electron cloud. One may also use vacuum chamber materials with low secondary electron yield or wrap the vacuum chamber with windings to generate solenoidal magnetic fields to suppress the generation of secondary electrons. These solutions are effective but expensive.

In another class of applications, the proton beams in high power proton drivers such as the Los Alamos Proton Storage Ring (PSR) have a relatively long bunch length with the result that the characteristic wavelength of the electron cloud 
instability in the proton bunch is normally much shorter than the bunch length. For this situation, manipulating the bunch spacing is not very practical and there are fewer effective strategies to combat the onset of the electron cloud instability.

The Proton Storage Ring (PSR) at the Los Alamos National Laboratory (LANL), where the experiments reported here were performed, is an $800 \mathrm{MeV}$ accumulator ring used primarily to provide $100 \mathrm{~kW}$ of beam for the spallation neutron source at the Los Alamos Neutron Science Center (LANSCE). For this use, PSR delivers $6.25 \mu \mathrm{C}$ pulses, about $270 \mathrm{~ns}$ wide at the current pulse base at a repetition rate of $20 \mathrm{~Hz}$.

The electron cloud instability, also known as the e-p instability, has been observed at the $\mathrm{PSR}^{6}$ since its commissioning in 1985. In this instability, the low-energy electron cloud is confined within the space-charge potential of the circulating proton beam. Coupled transverse oscillations of the beam and the trapped electrons ensue, which can lead to unstable, coherent motion of the beam and possible beam loss. Conditions for the instability are a copious source of low-energy electrons, a beam potential that can trap sufficient electrons, and coupled oscillations that are strong enough to overcome Landau damping mechanisms. A considerable body of evidence points to beam-induced multipacting, which is seeded by electrons from grazing angle beam losses, as the dominate source of electrons driving the e-p instability at the PSR. ${ }^{7-9}$ For long-bunch proton beams the multipacting occurs on the trailing edge of the beam bunch, hence the designation "trailing-edge multipactor."

The e-p instability has two significant distinctions from instabilities driven by conventional impedance in that the instability growth rates are typically much faster and have more pulse-to-pulse variability than those of conventional instabilities. The variability in growth rates is likely caused by the highly variable electron cloud density, which depends upon the pulse-to-pulse details of beam losses and trailingedge multipacting.

Prior to our tests of a prototype feedback system to be addressed in this paper, control of the PSR e-p instability was accomplished by various measures to enhance Landau damping including higher momentum spread from both higher RF buncher voltage and inductive inserts, use of multipole magnets, and coupled Landau damping using a skew quadrupole. $^{7-9}$ In addition, beam scrubbing during 2-3 years of increasing beam intensity has significantly improved the instability threshold, as shown in Fig. 1, where historical data on the instability threshold intensity are plotted versus the RF buncher voltage at the instability threshold.

Our interest in the use of transverse feedback as another means of mitigation is motivated by several factors:

- Active damping has been used effectively for many years to control a variety of transverse instabilities at other accelerators and storage rings.

- It employs a significantly different mechanism of control with limiting factors that are expected to be uncorrelated with those limiting Landau damping or electron cloud suppression.

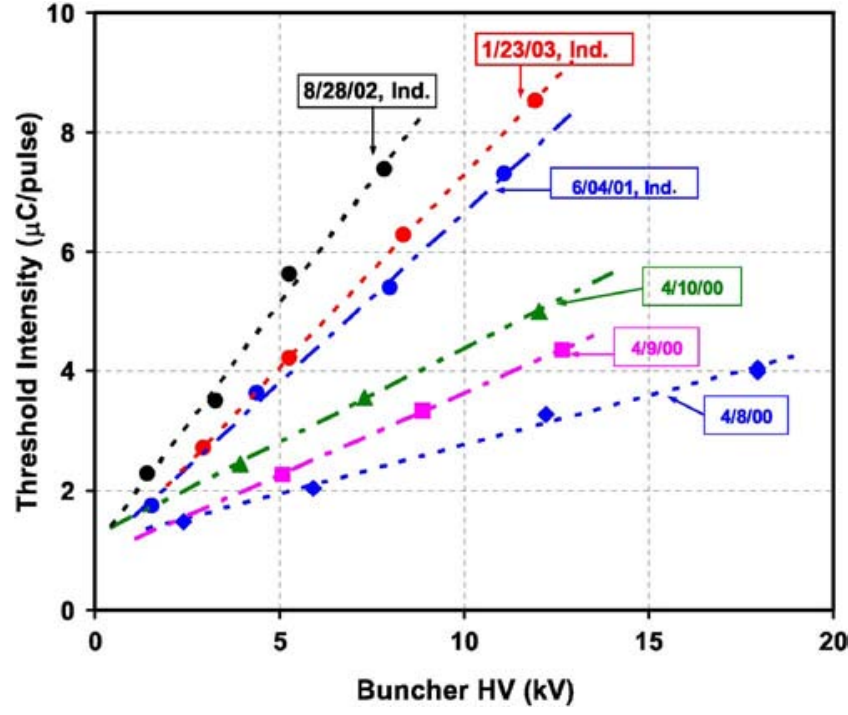

FIG. 1. (Color online) Instability threshold curves over a 3 year period of operation at increasingly higher beam intensity after the Direct $\mathrm{H}^{-}$Injection upgrade of 1998-1999. "Ind." refers to data with inductive inserts, which by themselves produce a $30 \%-40 \%$ improvement.

- It has the potential of control without increasing beam losses in the ring or downstream transport, unlike the measures to increase Landau damping which generally resulted in nonlinear changes in beam losses in the PSR.

- The e-p instability is a technical risk for the higher intensity proton accumulator rings such as the Spallation Neutron Source ring and future high power proton drivers. An experimental test of the concept for e-p is highly desirable in advance of any decision to implement such a system for routine operational use.

- While the present methods of control are adequate for most PSR operations, there is at least one application where additional measures are needed, namely for the high peak intensity, small emittance, single pulse beams that stimulate the so called "first pulse" e-p instability, in which the first pulse after a long wait is significantly more unstable than subsequent pulses. ${ }^{9,10}$

A feedback system for the e-p instability at PSR faces several major challenges, in addition to cost, that held off earlier implementation and warrant testing before a major investment is made. These include a fast growth rate (about 25-100 $\mu \mathrm{s}$ growth time), a broad bandwidth (50 $-300 \mathrm{MHz}$ ), a central frequency that depends primarily on the space charge density of the beam, and a driving mechanism, caused by the electron cloud, which is more variable and more uncertain than the driving forces for instabilities caused by structural impedance in the accelerator. With this background in mind, the main goal of this collaborative effort was to experimentally investigate the feasibility and effectiveness of feedback damping of the e-p instability, with a relatively modest investment in new hardware.

This paper is organized as follows. In Sec. II, we discuss the feedback system design. Experimental results carried out in 2005-2006 are reported in Sec. III, where we tested the feedback system and study the instability growth rate and the 


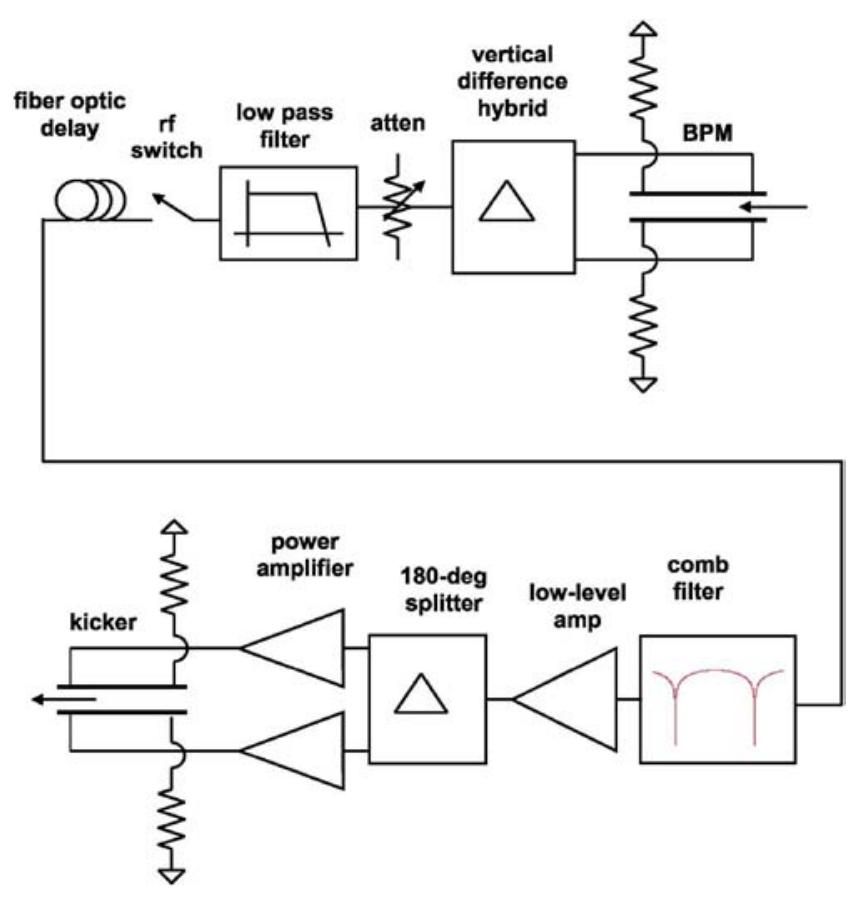

FIG. 2. (Color online) Block diagram of the prototype damping system tested at the PSR. Beam direction is right to left.

feedback damping rate. Discussion of some experimental observations is addressed in Sec. IV. Future directions in improving the e-p feedback system are discussed in Sec. V. The conclusion is given in Sec. VI.

\section{FEEDBACK SYSTEM DESIGN}

Planning and design of a prototype feedback system for this study began in the summer of 2004. Since the instability at PSR occurs in the vertical plane under most circumstances, the prototype was designed for feedback in that plane only, in order to keep costs to a minimum. However, it was recognized from the beginning that after a significant improvement in the threshold from feedback (estimated to be $30 \%-50 \%$ from a coasting beam model), the instability could show up in the horizontal plane. This was one of the issues we planned to address in our experimental tests of the prototype.

A simplified block diagram of the main components of the prototype system, in its final form, is shown in Fig. 2. A preexisting, short, strip-line beam position monitor (BPM) is used as the pickup to sense coherent motion from the instability. The vertical difference signal is formed with a suitable hybrid, then sent through a variable attenuator to adjust the gain and then to a low pass filter. A RF switch is used for fast gating of the feedback signal. The remainder of the low level RF system (LLRF) consists of a fiber optic delay, a comb filter that can be configured in a number of ways (including being by passed), a low level amplifier, and a 180 deg splitter. The split signal is sent to two $100 \mathrm{~W}$ RF amplifiers (ENI model 5100L-1431) and then by coaxial cable to the downstream end of each strip line kicker plate. The upstream end of each kicker plate was terminated in a $30 \mathrm{~dB}$ attenuator load (50 $\Omega$ ), whose output could be monitored or recorded on digital oscilloscopes. Various test points and monitor functions are not shown.

The BPM and the kicker are close to one another in Section 4 of the ring, with the BPM center upstream of the kicker center by $0.76 \mathrm{~m}$. For the standard betatron tunes of the ring, the vertical beta functions are calculated in MAD to be 7.5 and $9.7 \mathrm{~m}$ at the location of the BPM and kicker, respectively.

The damping rate of an ideal feedback system is given by

$$
\alpha_{o p t}=\frac{f_{0}}{2} G \sin \Psi_{p-k}
$$

where $f_{0}$ is the ring revolution frequency, $G$ $=\sqrt{\beta_{k} \beta_{p}} \cdot(d \theta / d y)$ is the feedback gain, $\beta_{k}$ and $\beta_{p}$ are the beta functions at the kicker and pickup, respectively, $d \theta / d y$ is the angular kick to the beam per detected vertical offset, and $\Psi_{p-k}$ is the relative betatron phase advance between the pickup and kicker. ${ }^{11}$ If we assume $\Psi_{p-k}=90 \mathrm{deg}$, and the gain is set so that the power amplifier outputs its maximum power $P$, when the vertical oscillation amplitude is at an amplitude $\Delta y_{\max }$, then the damping rate is given by

$$
\alpha_{s a t}=\frac{f_{0} \sqrt{\beta_{k} \beta_{p}}}{2 \Delta y_{\max }} \frac{e}{E \beta^{2}} \sqrt{2 P R_{\perp}},
$$

where $\beta$ is the velocity relative to the speed of light, $e$ is the charge of the electron, $E$ is the beam energy, and $R_{\perp}$ is the transverse shunt impedance of the kicker. For $N$ identical kickers each optimally located and receiving the same power, $P$, the damping rate will be $N$ times the right hand side of Eq. (2). In terms of the total installed feedback power, $P_{T}=N P$, the damping rate becomes

$$
\alpha_{s a t}=\frac{f_{0} \sqrt{\beta_{k} \beta_{p}}}{2 \Delta y_{\max }} \frac{e}{E \beta^{2}} \sqrt{2 N P_{T} R_{\perp}} .
$$

Note that $N$ kickers give a damping rate that is $\sqrt{N}$ times larger than for the same installed power applied to one kicker.

For a stripline kicker, we used a preexisting device that was designed as a BPM. A cross-sectional sketch is shown in Fig. 3 (top). The curved plates are at a radius of $51 \mathrm{~mm}$ inside a beam pipe with a $76 \mathrm{~mm}$ radius. Each curved plate subtends an angle of $114 \mathrm{deg}$ and has physical length of 0.37 m. Numerical calculations give a value of $48 \Omega$ for the impedance of the strip lines. An approximate expression for the effective shunt impedance, which assumes a plane wave propagating between parallel plates, is given by

$$
R_{\perp}=2 Z_{L}\left(\frac{2 g_{\perp} c \beta}{\omega d}\right)^{2} \sin ^{2}\left(\frac{\omega L(1+\beta)}{2 c \beta}\right),
$$

where $Z_{L}$ is the termination load impedance, $L$ is the length of the stripline plate, $d$ is the plate separation, $\omega$ is the angular frequency, $\beta$ is the beam velocity divided by the velocity of light, and $g_{\perp}$ is a geometric factor of order unity. A graph of the shunt impedance, calculated using Eq. (4) for $L=0.37 \mathrm{~m}$, is plotted as a function of frequency in Fig. 3 (bottom). 

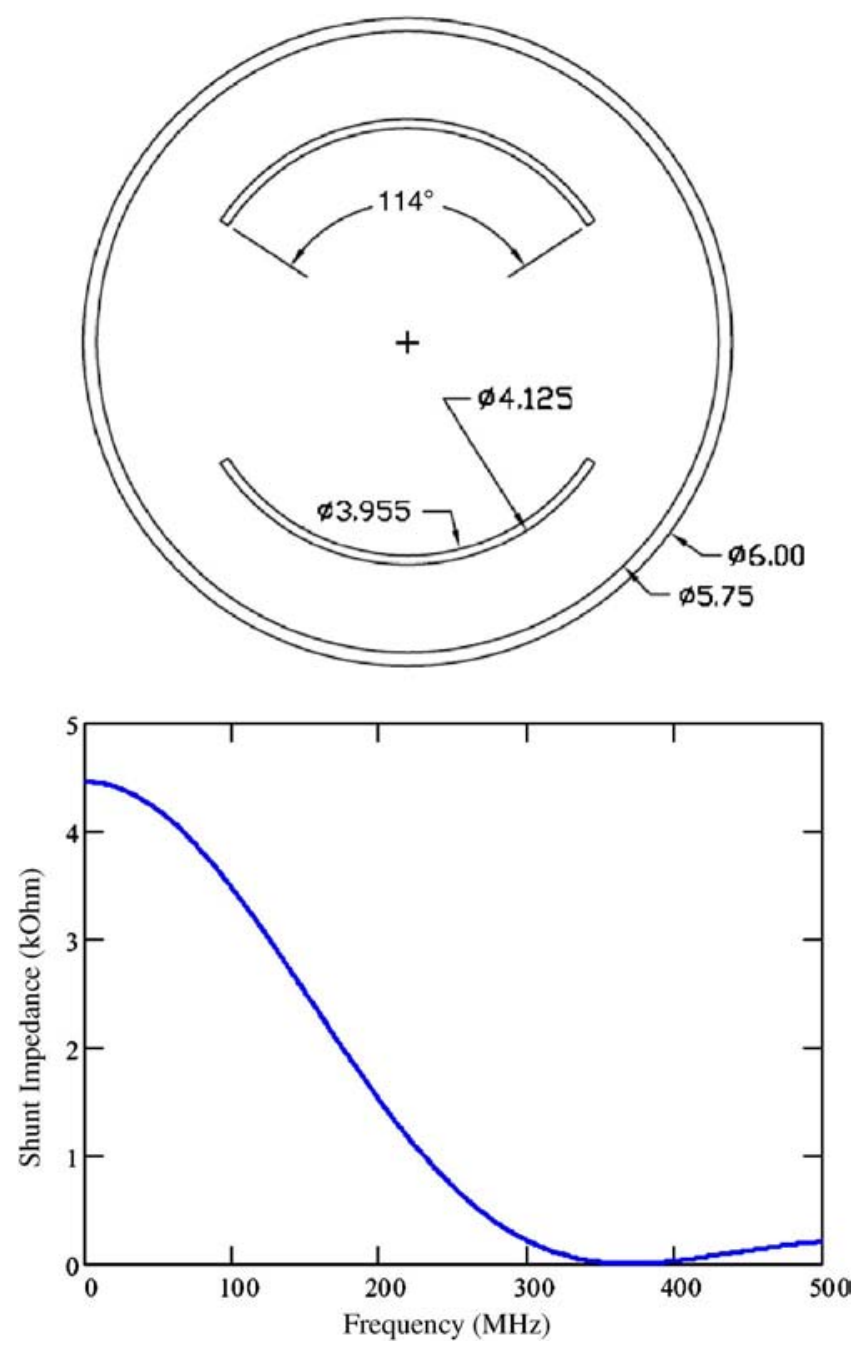

FIG. 3. (Color online) (Top) Cross-section of kicker. Dimensions are inches and degrees. (Bottom) Theoretical transverse shunt impedance vs frequency for the PSR kicker.

Assuming a maximum vertical oscillation amplitude of $0.5 \mathrm{~mm}$ at full feedback power, we can use Eqs. (2) and (4) to estimate the damping rate achievable with this system as about $30 / \mathrm{ms}$. As we will show later in Sec. III C, this is about the same as the vertical growth rates measured in the PSR near the instability threshold.

For the nominal vertical betatron tune of $Q_{y}=2.19$, a delay of four turns is optimally needed between the BPM output signal and the feedback waveform at the kicker, in order to produce an odd integral number times 90 deg of betatron phase advance for optimal damping. A comb filter section was added later in order to suppress revolution harmonics in the feedback waveform. This filter was a modular design that used fiber optic delays; it could be configured as one or two comb filters in series and each filter could be set independently with one or two turns of delay. ${ }^{12}$

It is of critical importance that the feedback system gain and phase allow for damping over the entire bandwidth of the unstable motion. For our system, the primary limitation in the bandwidth is set by the strip line kicker. The S12 frequency response of the LLRF, the comb filters, the final amplifiers, and the kicker were measured with a network
TABLE I. PSR and feedback parameters.

\begin{tabular}{lcc}
\hline \hline Parameter & Description & Value \\
\hline$E$ & Beam energy & $0.8 \mathrm{GeV}$ \\
$C$ & Circumference & $90.26 \mathrm{~m}$ \\
$f_{r f}$ & $\mathrm{RF}$ & $2.8 \mathrm{MHz}$ \\
$h$ & Harmonic number & 1 \\
$Q_{x, y}$ & Betatron tunes $(x, y)$ & $2.19,3.19$ \\
$P_{a m p}$ & Amplifier power & $2 \times 100 \mathrm{~W}$ \\
$\alpha_{\max }$ & Max damping rate & $30 / \mathrm{ms}$ \\
\hline
\end{tabular}

analyzer for signals that did not saturate the components. The measured bandwidth agrees with or is somewhat better than the calculated bandwidth, shown in Fig. 3 (bottom). A table of the PSR and feedback system parameters is given in Table I.

\section{EXPERIMENTAL RESULTS}

In this section we discuss results of tests of the system that were conducted during four blocks of dedicated beam development beam time at the PSR and tests carried out parasitically on beam production time for the user program during the 2005 LANSCE run cycle.

\section{A. Setup of the feedback system}

An important step in the setup of the damping system was the initial adjustment and optimization of the four turns of delay (no comb filter) between pickup and kicker. Several methods of adjustment were tried. These typically used the delay between distinctive features of the BPM signal for a short beam pulse (20 ns) and the same feature on the voltage waveform applied to the kicker and observed at the upstream end of the kicker. The delays obtained by these methods were consistent with the sum of electrical delays of the various subsystems measured by a network analyzer.

Once the initial delay adjustment was set to a fraction of the 5-8 ns period for the central frequency of the instability, the next step was to scan the delay in small (subnanosecond) increments to find the edges of the phase acceptance for good damping of the instability. The final setting was chosen to be half way between the edges. Finally, the overall gain was adjusted to produce the maximum improvement in the instability threshold.

\section{B. Observation of active damping}

The earliest and simplest evidence of damping of the e-p instability is shown in Fig. 4. With feedback off for a given beam intensity, the RF buncher voltage in the ring was lowered until the instability threshold was reached. At this point, there was significant exponential growth of coherent vertical motion of the beam centroid as shown by the red trace in Fig. 4. Although not indicated in this figure, the growth was accompanied by significant beam loss on about half of the beam pulses. When the feedback was turned on, the unstable motion would disappear as shown by the blue trace in Fig. 4. While the vertical coordinates in Fig. 4 are expressed in arbitrary units (A.U.), the display scale factor for the two BPM 


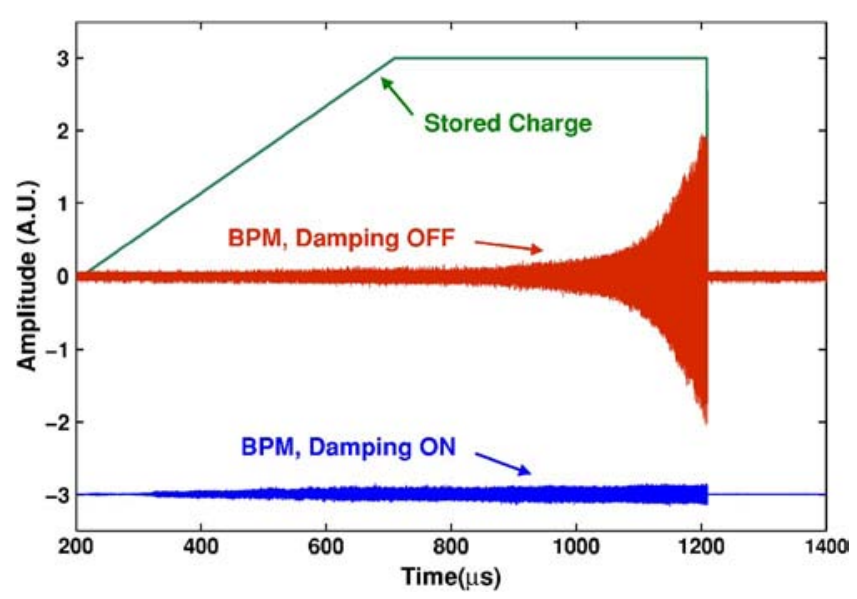

FIG. 4. (Color online) BPM vertical difference signal (red trace) at the instability threshold with feedback off compared to the BPM signal (blue trace) when feedback was on. The green trace is the circulating beam intensity in the ring, which is shown to indicate the accumulation and storage times. While the vertical coordinates are in arbitrary units (A.U.), the scale factor for the two BPM signals is the same.

signals is the same, and thus the change in amplitude between feedback on and off is accurately shown in this graph.

Our principal quantitative measure of effectiveness of the damping system is the change in instability threshold with feedback as compared with the value when feedback is off. A plot of threshold buncher voltage (for a fixed beam intensity) as a function of the relative voltage gain of the feedback system is shown in Fig. 5. Since it is easier to change the buncher voltage than the beam current, we use variation of the buncher voltage to find the threshold. The two variables are essentially equivalent as demonstrated by the well established linear relationship between the two shown in Fig. 1. The error bars of about 4\%-5\% in Fig. 5 are estimates based on our experience, over several years, with the short term reproducibility of the threshold measurements. They are also consistent with the threshold reproducibility observed during our experiments in the 2005 run cycle. From the curves of Fig. 5, we observe a maximum improvement in instability threshold voltage of $25 \%-30 \%$ between the threshold with feedback off (relative voltage

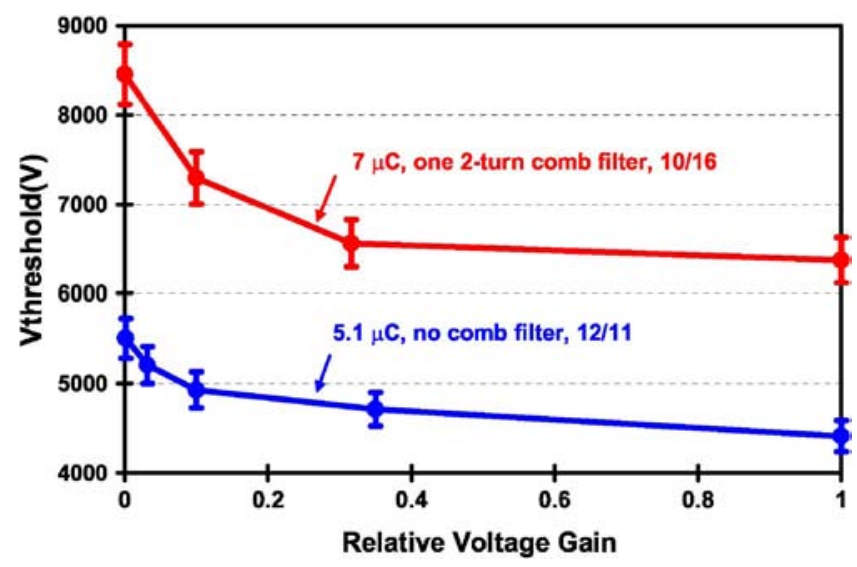

FIG. 5. (Color online) Instability threshold (RF buncher voltage) as a function of relative gain of the feedback system for two beam intensities, $7 \mu \mathrm{C}$ /pulse (red trace) and $5.1 \mu \mathrm{C}$ /pulse (blue trace). gain of 0) and the threshold with feedback on at the highest gain (relative voltage gain of 1). We were not able to further improve upon this result and thus devoted much effort to identify and analyze the factors limiting the improvement.

\section{Other damping observations}

The results in Fig. 5 also show there was little, if any, additional improvement using a comb filter. Several filter combinations were tried with similar results. Introduction of a closed orbit bump of several $\mathrm{mm}$ at the location of the pickup had little effect on the damping system as measured by the change in instability threshold. These observations suggest that diversion of feedback power to closed orbit offset is not the dominant factor limiting the feedback effectiveness.

The effect of feedback on smaller emittance beams was also studied, since smaller emittance beams have higher space charge fields for the same given stored charge and are known from previous work to be more unstable. In addition, the bounce frequency of electrons is higher and the growth rate of the instability generally higher. The effect of smaller emittance was studied on two occasions when a beam was prepared using a smaller injection offset that fills a smaller transverse emittance area. For these beams, feedback improved the instability threshold voltage by only $10 \%-18 \%$, about half the improvement observed with larger emittance beams of the same total stored charge.

We should also note that transverse feedback introduces the potential risk of emittance growth caused by noise in the feedback waveform. Beam spot size and beam loss measurements for stable beams were made with feedback off and compared with those with feedback on; the data showed no measurable increase in beam emittance or beam loss using feedback.

\section{Measurement of growth and damping rates}

Before the installation of the prototype feedback system, we could only measure growth rates at the instability threshold. With the prototype system, we can turn feedback on or off anytime during the cycle by gating the RF switch in the LLRF using a suitable wave form. This provides the capability for measurement of the instability growth rates during the growth phase and measurement of the damping rates with feedback on in the damp phase. Furthermore, with dampgrow-damp experiments there is the possibility to measure instability growth rates above the instability threshold, referred to simply as a grow/damp measurement. Grow/damp experiments were performed for conditions that produce an unstable beam with feedback off (i.e., a buncher voltage significantly below the threshold voltage for a given stored charge).

Results from one such experiment using a $7 \mu \mathrm{C} /$ pulse beam are shown in Figs. 6-8. The unstable motion (blue trace) shown in Fig. 6 started to grow with a characteristic exponential growth after feedback was turned off and then damped when the feedback was gated on again at the end of 


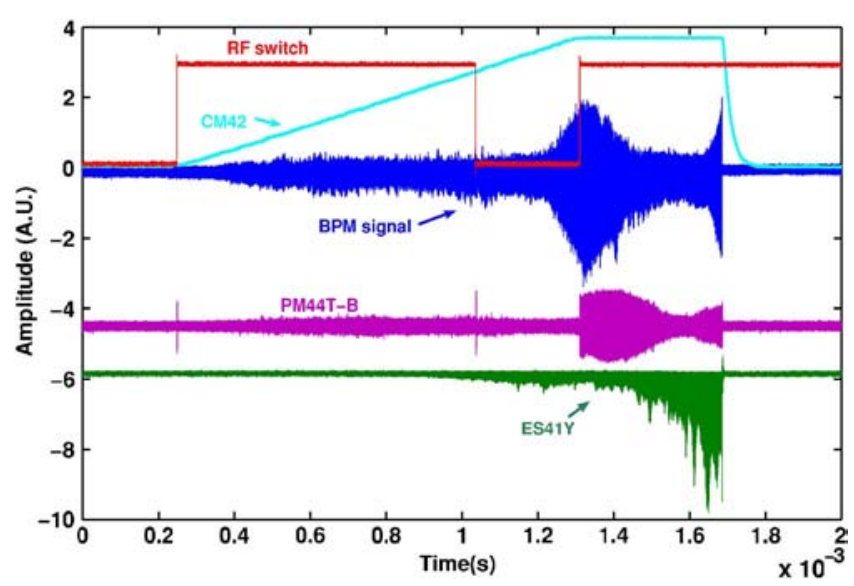

FIG. 6. (Color online) Plot of simultaneous signals (arbitrary units) from a damp-grow-damp experiment. In the top portion of the graph, the RF switch signal (red) is shown along with the BPM vertical difference signal (blue) and a beam current monitor signal (CM42, cyan trace). Offset below these signals are the vertical difference signal from the upstream end of the kicker (PM44T-B, magenta trace) and an electron cloud signal from ES41Y (green trace).

accumulation. The second period of growth, which shows up at the end of the storage time just before extraction, will be discussed later.

There are several ways to define growth or damping rates and a number of methods to analyze the BPM signal for these rates. For example, one can measure the growth of the amplitude envelope, or of the rms amplitude, or growth of a particular mode. We have chosen to calculate the power
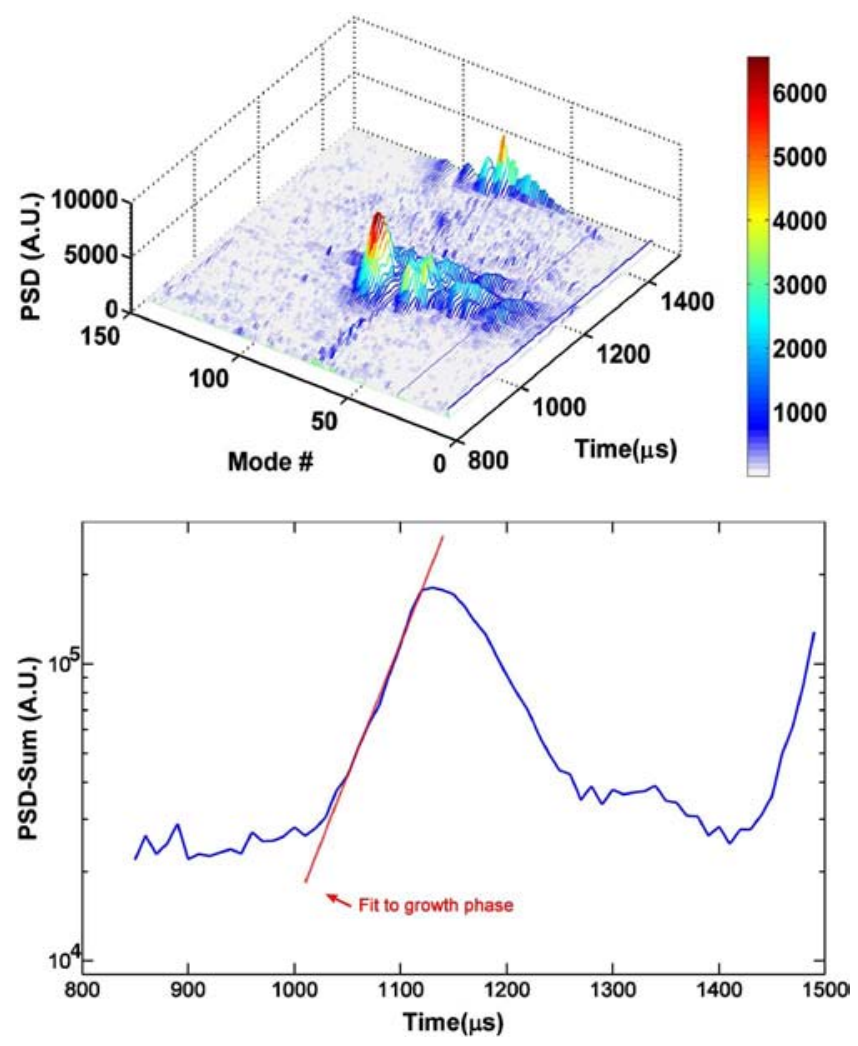

FIG. 7. (Color online) (Top) PSD spectrogram of BPM signal shown in Fig. 6. (Bottom) Log of PSD summed over modes 25-125. Amplitude growth time is $98 \mu \mathrm{s}$. The vertical scales in both graphs are in arbitrary units (A.U.).

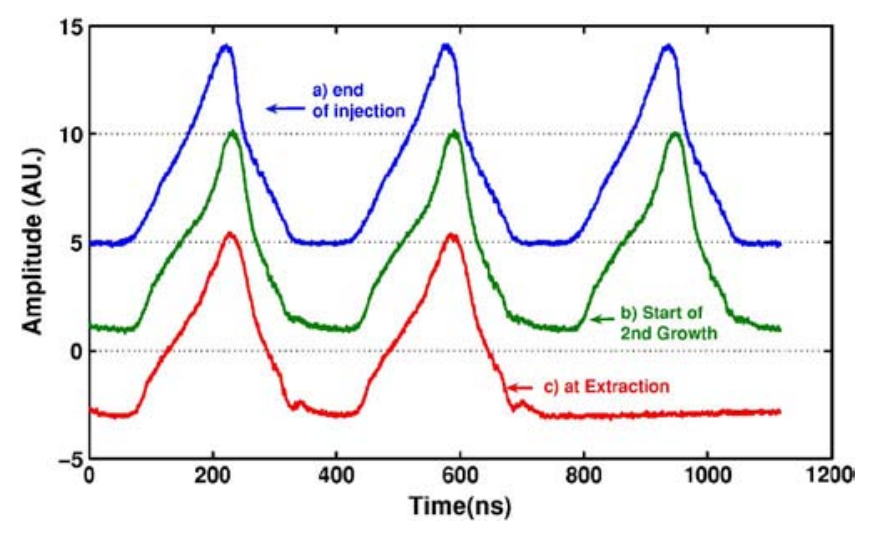

FIG. 8. (Color online) Plot showing a turn-by-turn expansion of the signal (arbitrary units) from a wall current monitor (WC41) at three different times in the beam pulse for the damp-grow-damp experiment. The blue trace (a) starts at the end of injection, the green trace (b) at the start of second growth period, while the red trace (c) shows the last two turns before extraction.

spectral density (PSD) and sum over a band of modes. The PSD is calculated from the square of the FFT (fast Fourier transform) amplitude using non-overlapping, rectangular time windows and plotted as a function of time, as shown in the top of Fig. 7. The PSD is calculated with a $10 \mu$ s time window, sufficient to separate betatron side bands from revolution harmonics. We obtained growth and damping rates from PSD spectra by summing over a band of modes that captures the unstable motion. The log of the PSD sum is plotted as a function of time in the bottom of Fig. 7 and the slopes of growth and damping regions were measured.

The exponential growth and damping rates for power obtained from the plot of Fig. 7 are $20.6 / \mathrm{ms},-14.4 / \mathrm{ms}$, and $31.9 / \mathrm{ms}$ for the first growth region, the damping region, and the second growth region, respectively. If we assume that the damping system was operating in the linear region, then the appearance of the second growth region implies that the underlying instability growth rate exceeds the damping rate. From this we would infer a growth rate of a factor of about 3 higher than the growth rate for the first growth region. Examination of the feedback signals showed that the amplifier output was proportional to the beam pickup signal, indicating that the feedback was not saturated and, therefore, in the linear regime.

We offer two basic scenarios or hypotheses to explain the large change in instability growth rate during $400 \mu$ s of storage at constant beam intensity. In many models for the e-p instability, e.g., those in Refs. 13 and 14, the instability growth rate depends on the density of electrons oscillating in the beam potential. This insight suggests a scenario where significantly more electrons are generated during the store, survive the gap, and are captured by the beam to drive the instability with greater strength. In the second scenario, the momentum spread of the beam changes as a longitudinally mismatched beam undergoes synchrotron motion, thereby causing a change in Landau damping rate.

There is evidence of a significant increase in the electron cloud during the second damping region of Fig. 6. The green trace labeled ES41Y in Fig. 6 is the electron cloud signal from a retarding field analyzer (RFA) type electron detector signal $^{15}$ located in a drift space. This device provides a mea- 
sure of the electrons striking the wall during trailing-edge multipactor, and in Fig. 6 the electron signal shows a factor of 5 or more increase over the $400 \mu$ s storage time. While such evidence is suggestive, it is not conclusive proof that more electrons survived the gap to be captured by the next beam pulse, since previous studies in 2001 (Ref. 9) have shown that electrons surviving a clean gap saturate at these intensities. However, there has been continual beam scrubbing since then, so the intensity for saturation could have changed to a higher intensity.

If there is some beam-in-the-gap, then more of the multipacting electrons will survive the gap and be captured by the next pulse. Previous unpublished studies in 2001 and 2002, when controlled amounts of beam were introduced in the gap, demonstrated a linear relation between electrons surviving the gap and the amount of beam-in-the-gap. In these studies, adding a certain amount of beam-in-the-gap caused an increase in the number of electrons surviving the gap by an amount that neutralized the beam-in-the-gap.

Some evidence for beam-in-the-gap, in the grow/damp experiment discussed above, is shown in Fig. 8, which is a plot of the wall current monitor signal at three stages of the beam storage. There is little beam-in-the-gap at the end of injection when the feedback was turned on. By the start of the second instability growth phase there is evidence of beam leakage into the gap with a level of about $2 \%$ of the peak current. This level would be roughly consistent with a factor of about 3 increase in the growth rate in the delta function model of Channell. ${ }^{13}$ The lower buncher voltage, made possible with feedback, can cause beam leakage into the gap and points to a possible explanation for the limited improvement in instability threshold with feedback. With feedback on we lower the buncher voltage to find the new instability threshold and, in doing so, create more beam-in-the-gap. This was certainly exacerbated by poor control of the energy of the beam from the linac, as a result of problems with some of the linac power amplifiers during the 2005 run cycle.

Other features of note in the progression shown in Fig. 8 relate to the changes in the shape of the longitudinal profile of the beam during the store time. During the typical PSR accumulation time there are only one to two synchrotron periods (about $500 \mu \mathrm{s}$ ). Therefore, the beam is not, in general, particularly well matched in longitudinal phase space, and the longitudinal profile can change during the store time as the beam rotates in longitudinal phase space. In addition, the bunch width is a large fraction of the bucket width, and this leads to a significantly lower synchrotron frequency for the protons injected near the ends of the bunch. The changes in pulse shape during the store can also have a strong impact on the electron cloud generation by the trailing edge multipactor and may explain the strong increase in the electron detector signal during the store, as shown in Fig. 6. The effect of the observed changes in longitudinal profile on the electron cloud generation could be simulated in a code such as POSINST (Ref. 17) using the measured profiles, but we have not yet been able to devote the computational resources needed for this task.

\section{DISCUSSION}

Much effort during the last half of the 2005 run cycle was devoted to identification and analysis of the factors limiting the improvement in instability threshold with feedback. A number of issues were addressed including

- factors that increase the instability growth rate including electron cloud formation, beam-in-the-gap, and beam parameters such as the beam emittance;

- the role of instabilities in the horizontal plane; and

- feedback system issues including system gain, bandwidth, nonlinearities, and saturation behavior plus noise power in revolution harmonics.

Design of a feedback system requires knowledge of the range of frequencies to be covered and the range of growth rates to be damped. For the e-p instability at the PSR, the frequency range of the instability is related to the range of bounce frequencies of electrons in the space charge potential of the beam. This relationship is well established by both basic theory and observation, once the basic beam parameters are known. However, the same cannot be said for the growth rates and the factors that influence them. In previous studies, we observed that the instability at PSR is fast, with amplitude growth times at threshold in the range of 25-100 $\mu$ s. The prototype feedback system was designed to deal with the more typical $75 \mu$ s amplitude growth time. Since the feedback power requirements (hence costs) scale as the square of the highest growth rate to be damped, the maximum instability growth rate needs to be better determined for cost-effective, future improvements to a feedback system at the PSR.

\section{A. Growth rates}

Theoretical estimates of growth rates are rather uncertain with most analytical theories predicting much faster growth rates than we typically observe at PSR. Experimental data on growth rates are rather limited at present. More detailed studies are needed to identify the important parameters affecting them and to measure their variation over a suitable range of parameter changes.

Results of the damp-grow-damp experiments, discussed in the previous section, provide some insight into one of the sources (beam-in-the-gap) of variability in growth rates, and how it would limit the performance of the feedback system. Based on our experience to date, we would not recommend reliance on feedback to overcome the higher growth rate from beam-in-the-gap, since a clean gap is also needed to minimize beam loss during extraction. A smaller injected bunch width is also desirable, but comes at the expense of longer accumulation (with more beam losses) or a higher source intensity, in order maintain the same accumulated charge.

Previous studies and some of the recent tests of the feedback system with smaller emittance beams, but with the same accumulated charge (higher space charge density), have shown faster growth rates as well as lower thresholds. We have also seen more variability in the instability growth 


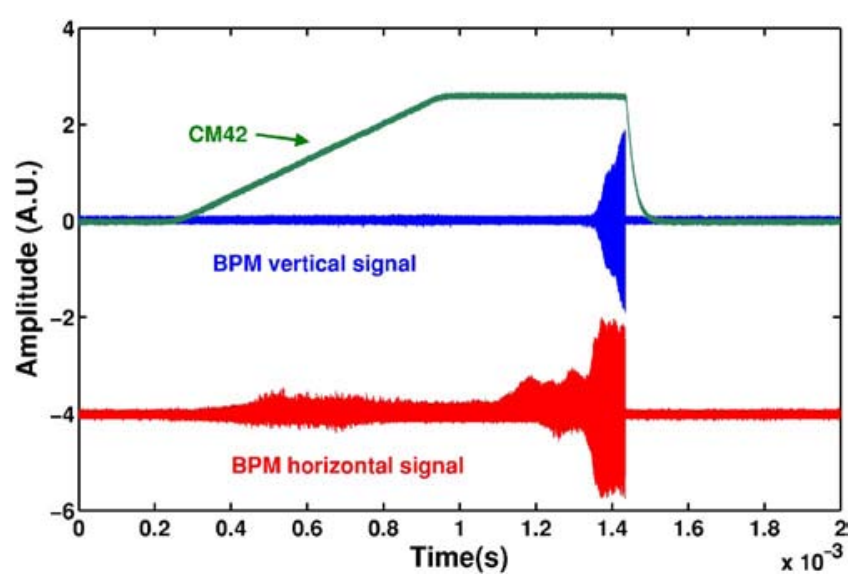

FIG. 9. (Color online) Vertical difference signal (blue) from the BPM and horizontal difference signal (red) from the 30 June 2005 test. A signal from the beam current monitor (green) in the ring is also shown to indicate the accumulation and store times. Vertical scales are in arbitrary units; however, the horizontal BPM signal has a display gain that is a factor of 3.5 higher than the vertical.

rate for the smaller emittance beams, but the details of the dependence on space charge density have not yet been studied systematically.

\section{B. Instability in the horizontal plane}

Since we only feed back in the vertical plane it was estimated that, after about 30\%-50\% improvement in the vertical threshold, the instability might appear in the horizontal plane. On at least one occasion with feedback on and a charge of $5.1 \mu \mathrm{C}$, we recorded the appearance of the instability in the horizontal plane before it was observed in the vertical, as is shown in Fig. 9. In this experiment, the feedback was on and the RF buncher voltage lowered to the threshold of the instability (30\% lower voltage). For these conditions, unstable coherent motion first appeared in the horizontal plane.

It is not surprising that the instability eventually shows up in the horizontal plane when the vertical is sufficiently damped by feedback. In the simple coasting beam theory with Landau damping, the threshold is proportional to the betatron tune, $Q$; hence, in the absence of feedback and all other parameters being equal, the vertical is more unstable by virtue of the smaller $Q_{y}=2.19$ compared to $Q_{x}=3.19$. However, with feedback stabilizing the vertical motion, the horizontal plane will become unstable as the buncher voltage is lowered and the Landau damping is insufficient to overcome the horizontal growth rate. Therefore, in this simple model, one expects the horizontal to become unstable at a buncher voltage that is about $30 \%$ lower, which is consistent with observations. The cause of the very fast growth in this setup may once again be due to beam-in-the-gap, which traps more of the electron cloud during passage of the gap. We also note that the evolution of the longitudinal profile of the bunch here is very similar to that of the damp-grow-damp experiment described in the previous section.

\section{Feedback system issues}

From an electrical engineering viewpoint, the prototype system performed as intended. The low-loss fiber optic delay lines were essential to obtaining the four turn delay. The comb filters had excellent electrical characteristics, with deep notches at the revolution harmonics. The main system concerns, from the beginning, were feedback gain/power and bandwidth. Output power of $100 \mathrm{~W}$ for each kicker plate was estimated to be adequate for growth times (in amplitude) of $75 \mu \mathrm{s}$ (200 turns) and centroid motion amplitude of $1 \mathrm{~mm}$ at full power, which are typical for 5-6 $\mu \mathrm{C}$ beams.

The measured bandwidth of 30 to $250 \mathrm{MHz}$ of the RF system would cover most of the observed frequency content for the instability at intensities up to $6 \mu \mathrm{C}$ /pulse for the standard beam emittance encountered in routine operations for the LANSCE spallation neutron source. In our beam tests of the prototype system, we saw no obvious evidence that system bandwidth was limiting performance, such as unstable motion outside the bandwidth. An exception might be the smaller emittance, high space charge beams, but these data await detailed analysis. The first pulse instability typically has higher frequencies and higher growth rates, but we did not have the opportunity to investigate the effectiveness of feedback, in a controlled way, for this situation.

The fast growth rates, encountered when the buncher voltage was lowered to the instability threshold with feedback on, were somewhat unexpected. Our studies, described in earlier sections, suggest that these are caused by beam leakage into the gap between bunches, such that many more electrons survive the gap to be captured by the next beam pulse and drive the instability. It is not advisable to design a feedback system to handle the high growth rates arising from beams with several percent beam-in-the-gap; such beams will typically have high extraction losses that should be avoided by adequate RF buncher voltage, preparation of an energy-stable beam from the linac, and careful setup of injection into the RF bucket to keep beam from leaking out of the bucket.

The special situations that give rise to the so called first pulse instability typically use the maximum voltage available from the RF buncher. We did have feedback on for one such run, but it was not enough to stabilize the beam. Feedback might be useful here; but more study is needed to pin down the highest growth rates that must be damped.

\section{FUTURE DIRECTIONS}

From the experience gained in these experiments we offer the following suggestions for improving the effectiveness of feedback in controlling the e-p instability at the PSR and similar long-bunch proton accelerators:

- Deploy feedback in both planes. In our tests, we observed that the instability threshold for the horizontal plane is within $30 \%$ or so of the threshold in the vertical.

- More power is needed, especially for beams with higher space charge density. We suggest the use of multiple kickers that are somewhat shorter than those used in the prototype. The upper frequency limit will 
be higher with shorter kickers; multiple kickers or distributed kickers will require less total power than a single kicker system with the same damping rate.

- Avoid conditions that induce beam-in-the-gap. Use sufficient RF voltage and somewhat smaller bunch widths for the injected beam. Correction of the anomalous energy droop from the linac, experienced during the 2005 run cycle, is also suggested to reduce the conditions that produce beam-in-the-gap.

- Directional couplers in the output lines would be useful for careful monitoring of the applied power, with minimal contamination by beam-induced signals from the kicker.

As mentioned before, we would not advise investing in a system powerful enough to cope with the growth rates arising from several percent of beam-in-the-gap. If possible, one should use enough RF buncher voltage to keep leakage into the gap to less than $1 \%$. A gap cleaning device, such as a suitable fast kicker and collimator, in combination with feedback could be effective, but the feasibility of this approach to gap cleaning at the PSR is uncertain and needs more study.

A better characterization, both theoretical and experimental, of the growth rates of the instability and the factors that influence them is clearly needed in order to properly design a feedback system. Experiments to measure e-p growth rates and thresholds as a function of beam-in-the-gap would provide additional insight into their dependence on the number of electrons captured by the beam.

\section{CONCLUSIONS}

The prototype e-p instability feedback system was successfully demonstrated at the PSR. We were able to use the grow-damp-grow experiments to confirm the nature of the e-p instability and the feedback system damping rate that agreed well with the expectation. We tested and found that comb filters did little to improve the damping rate at a constant amplifier power. The prototype feedback system was able to improve the instability threshold (as measured by the RF buncher voltage) by $30 \%$ and shows that the electron cloud instability for proton beams can be effectively controlled by implementing a broadband feedback system.

Further improvement appears to be limited mainly by two factors: (1) beam leakage into the gap between bunches brought on by the lower RF buncher voltage and abnormal energy variations (energy droop during the macropulse) of the beam injected into the ring, and (2) onset of the instability in the horizontal plane that did not have feedback.

Beam leakage into the gap significantly increases the number of electrons that survive the passage of the gap and are captured by the next beam pulse where they oscillate in the space charge field of the beam and drive the e-p instability. Instability growth rates, observed with feedback on and in the presence of significant beam-in-the-gap (several percent), are several times higher than those for unstable beams with a relatively clean gap and no feedback. The appearance of the instability in the horizontal plane, when the buncher voltage was lowered $30 \%$ with feedback on (only in the ver- tical plane), was anticipated from the analytical coastingbeam model for e-p. For maximum effectiveness, both planes should have feedback.

\section{ACKNOWLEDGMENTS}

We gratefully acknowledge the dedicated beam time and other support provide by the LANSCE division at LANL as well as numerous helpful discussions with Doug Gilpatrick, John Power, and Larry Rybarcyk. The authors would also like to acknowledge the splendid efforts of the operating crews at LANSCE, who made every effort to meet the beam requirements for these studies.

The LANL work up through 1 June 2006 was supported by the U.S. Department of Energy under Contract No. W-7405-ENG-36 (with the University of California) and after 1 June 2006 under Contract No. DE-AC52-06NA25396 (with the Los Alamos National Security, LLC). ORNL/SNS is managed by UT-Battelle, LLC, for the U.S. Department of Energy under Contract No. DE-AC05-00OR22725. The LBNL work was supported by the Director, Office of Science, of the U.S. Department of Energy under Contract No. DE-AC02-05CH11231. The Indiana University group is supported by grants from the U.S. Department of Energy under Contract No. DE-FG0292ER40747 and the National Science Foundation Grant No. NSF PHY-0552389.

${ }^{1}$ K. C. Harkay, Proceedings of 31st ICFA Beam Dynamics Workshop on Electron Cloud Effects, 19-23 April 2004, Napa, CA, CERN Report No. 2005-001, pp. 9-13.

${ }^{2}$ K. C. Harkay, Proc. 2006 European Particle Acceleration Conf., Edinburgh, Scotland, 2006, WEXFI02, pp. 1887-1891.

${ }^{3}$ A. Wolski, Proceedings of 31st ICFA Beam Dynamics Workshop on Electron Cloud Effects, 19-23 April 2004, Napa, CA, CERN Report No. 2005001, pp. 407-416.

${ }^{4}$ M. A. Furman, ICFA Beam Dyn. Newslett. 33, 70 (2004).

${ }^{5}$ R. C. Davidson and H. Qin, Physics of Intense Charged Particle Beams in High Energy Accelerators (World Scientific, Singapore, 2001), Chap. 10.4 .

${ }^{6}$ D. Neuffer, E. Colton, D. Fitzgerald, T. Hardek, R. Hutson, R. Macek, M. Plum, H. Thiessen, and T.-S. Wang, Nucl. Instrum. Methods Phys. Res. A 321, 1 (1992).

${ }^{7}$ R. J. Macek et al., Proc. PAC 1, 688 (2001).

${ }^{8}$ R. J. Macek, Proceedings of ECLOUD'02, 15-18 April 2002, CERN, Geneva, CERN Report No. 2002-001, pp. 259-268.

${ }^{9}$ R. J. Macek, A. A. Browman, M. J. Borden, D. H. Fitzgerald, R. C. McCrady, T. Spickermann, and T. J. Zaugg, Proceedings of 31st ICFA Beam Dynamics Workshop on Electron Cloud Effects, 19-23 April 2004, Napa, CA, CERN Report No. 2005-001, pp. 63-75.

${ }^{10}$ R. J. Macek, T. Spickermann, A. Browman, D. Fitzgerald, R. McCrady, and T. Zaugg, Proceedings of 39th ICFA Advance Beam Dynamics Workshop, HB2006, 29 May-2 June 2006, Tsukuba, Japan.

${ }^{11} \mathrm{~J}$. Rogers, Handbook of Accelerator Physics and Engineering, edited by A. W. Chao and M. Tigner (World Scientific, Singapore, 1998), p. 490.

${ }^{12}$ C. Deibele, S. Assadi, V. V. Danilov, S. Henderson, M. Plum, C. Sibley, S. Breitzmann, S-Y. Lee, J. D. Gilpatrick, R. J. Macek, R. C. McCrady, J. F. Power, T. Zaugg, and J. Byrd, Proc. 2006 European Particle Acceleration Conf., Edinburgh, Scotland, 2006, THPCH130.

${ }^{13}$ P. J. Channell, Phys. Rev. ST Accel. Beams 5, 114401 (2002).

${ }^{14}$ H. Qin, E. A. Startsev, and R. C. Davidson, Phys. Rev. ST Accel. Beams 6, 014401 (2003).

${ }^{15}$ R. J. Macek, A. Browman, M. Borden, D. Fitzgerald, T. Zaugg, K. Harkay, and R. Rosenberg, Proc. 2003 IEEE Particle Acceleration Conf., Portland, OR, 2003, p. 508.

${ }^{16}$ R. J. Macek, AIP Conf. Proc. 448, 116 (1998).

${ }^{17}$ M. T. F. Pivi and M. A. Furman, Phys. Rev. ST Accel. Beams 6, 034201 (2003). 